\title{
Particle Motion Analysis Reveals Nanoscale Bond Characteristics and Enhances Dynamic Range for Biosensing
}

\author{
Emiel W. A. Visser ${ }^{*,+, \neq}$, Leo J. van IJzendoorn ${ }^{\dagger, \ddagger}$, Menno W. J. Prins ${ }^{\dagger, \neq, \S}$ \\ ${ }^{\dagger}$ Department of Applied Physics, Eindhoven University of Technology (TU/e), Eindhoven, the Netherlands \\ ${ }^{\ddagger}$ Institute for Complex Molecular Systems (ICMS), TU/e, Eindhoven, The Netherlands \\ ${ }^{\S}$ Department of Biomedical Engineering, TU/e, Eindhoven, the Netherlands \\ *E-mail: e.w.a.visser@tue.nl
}

\section{Particle position tracking accuracy}

To determine the position tracking accuracy, particles were immobilized onto the glass substrate and their motion amplitude was determined. The particles were immobilized by baking a solution of particles onto the glass slides in an autoclave at $121^{\circ} \mathrm{C}$ for 20 minutes in which the particles bond with the substrate. The motion of the immobilized particles was then observed. The motion of an immobilized particle and the analysis of the ensemble of immobilized particles is shown in figure S1.
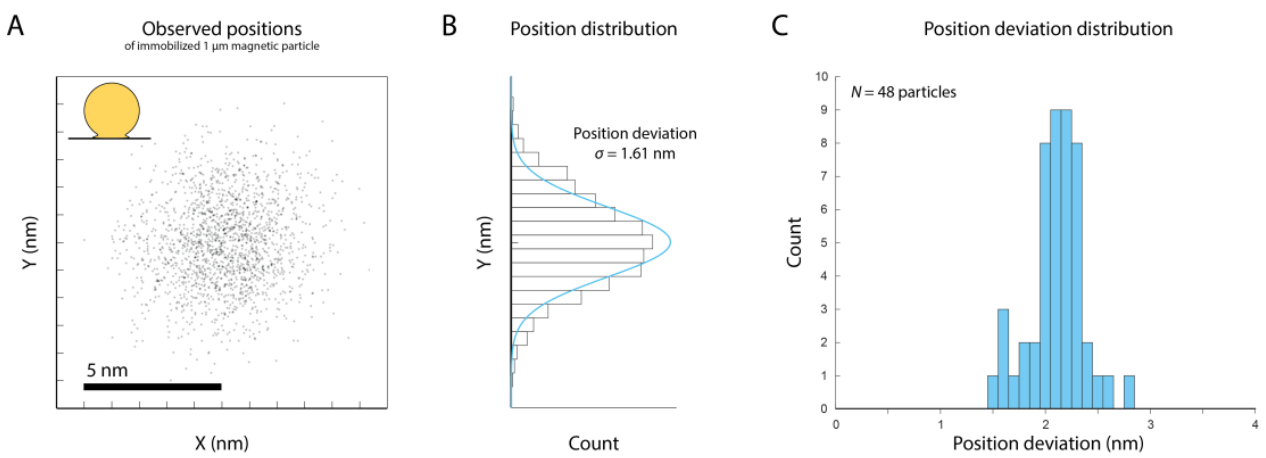

Figure S1: A) observed positions of an immobilized particle. Scalebar: $5 \mathrm{~nm}$. B) Position distribution along yaxis. The black shows the histogram of the observed positions, the blue line shows the normal distribution with $\sigma=1.61 \mathrm{~nm}$ determined from the histogram. C) distributions of $\sigma_{\text {position }}$ of 48 immobilized particles.

The observed positions of a single immobilized particle is shown in figure S1A. The position distribution along the $y$-axis of the observed positions is shown in figure S1B with a standard deviation of $1.61 \mathrm{~nm}$. The observed positions are determined by two contributions; the motion of the particle and the measurement error caused by for example signal noise and drift correction. By assuming that the particle is entirely immobilized, the maximum error in the position determination can be estimated as $\sigma_{\text {position }}$.

The distribution of 48 immobilized particles in terms of $\sigma_{\text {position }}$ is shown in figure S1C. The range of observed $\sigma_{\text {position }}$ is between approximately 1.5 and $2.8 \mathrm{~nm}$ with a most frequently observed $\sigma_{\text {position }}$ of approximately $2.25 \mathrm{~nm}$. We therefore estimate that the position error for this optical setup is $<3 \mathrm{~nm}$. 


\section{Quantification of DNA binding efficiency on the particles}

The efficiency of DNA binding to the particle was quantified using a supernatant assay. In the supernatant assay a known concentration of DNA strands was incubated with the particles for 60 minutes and finally using magnetic separation the particles were separated from the supernatant. The concentration of unbound DNA in the supernatant was determined and the bound fraction of DNA strands can be calculated.

The concentration of DNA was determined using PicoGreen (Life Technologies) as a DNA intercalating dye. PicoGreen absorbs blue light at $497 \mathrm{~nm}$ and after binding to DNA emits green light at $520 \mathrm{~nm}$. The fluorescent signal intensity was used to quantify the concentration of DNA in the sample.

MyOne particles were functionalized at particle to DNA ratio of 1:10. A volume of $50 \mu \mathrm{l}$ of $16 \mathrm{pM}$ MyOne particles suspended in TE buffer and $50 \mu \mathrm{l}$ of $160 \mathrm{pM}$ DNA strands in TE buffer was incubated for 60 minutes in a DNA lo-bind epp, after which the supernatant was extracted using magnetic separation.

Calibration concentrations of known DNA concentrations in TE buffer between 1.25 pM and 160 pM were prepared from the same solution used to functionalize the particles and mixed with PicoGreen at the suppliers recommended concentration. The fluorescence signal was measured in a Cary Eclipse fluorescence spectrometer from Agilent (Santa Clara, Ca, United States) at an excitation wavelength of $490 \mathrm{~nm}$ and an emission wavelength of $530 \mathrm{~nm}$. Both excitation and emission slits were set to $10 \mathrm{~nm}$ with no filters in place. A small volume quartz cuvette type 105.250-QS from Hellma Analytics (Müllheim, Germany) was used for the fluorescent measurements. The resulting measurements are shown in Figure $\$ 2$.

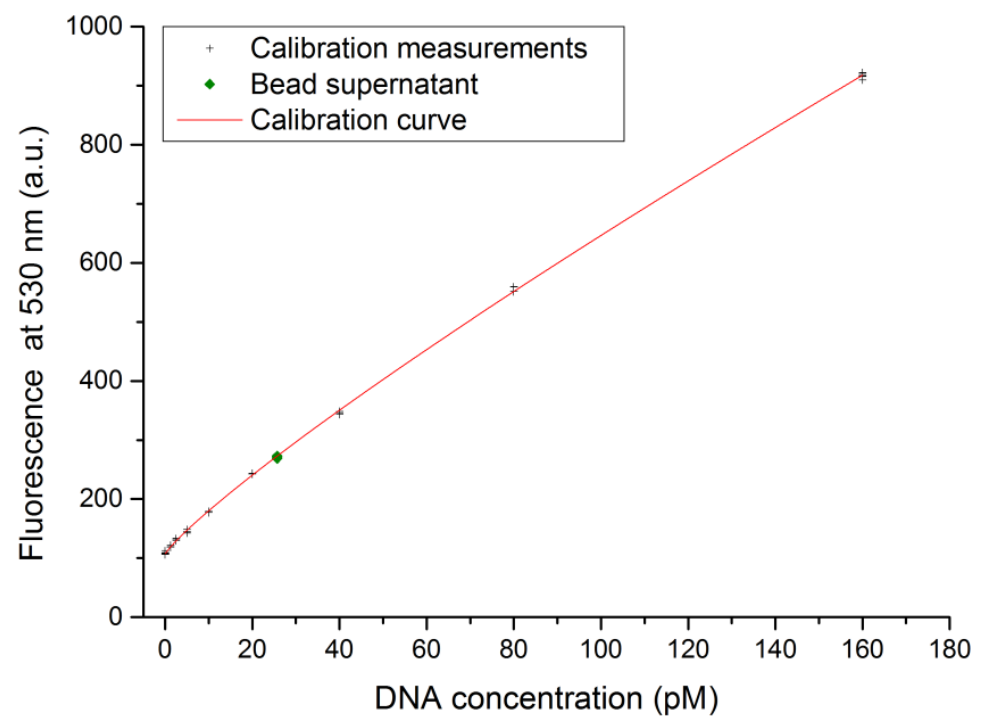

Figure S2: Fluorescence measurements of various concentrations of DNA in TE buffer using PicoGreen as DNA intercalating dye. Crosses indicate the calibration measurement points. The red line is a first order power law fit to the calibration data: $y=107.25+9.92|x-0.064|^{0.86753}$. The supernatant measurement is shown as a green dot at the corresponding calculated DNA concentration. 
The DNA concentration present in the supernatant is $25 \mathrm{pM}$. Taking into account that the original 50 $\mu \mathrm{l}$ was diluted to $100 \mu \mathrm{l}$ by mixing with the particle solution this indicates that $110 \mathrm{pM}$ of DNA has been extracted from the supernatant by the particles. This calculates to a DNA binding efficiency of $69 \%$.

\section{Expectation value of the number of DNA strands that can interact with the substrate}

The binding capacity of biotinylated DNA on the MyOne particles was specified at $500 \mathrm{pmol}$ of DNA fragments per $1 \mathrm{mg}$ of MyOne particles, which equates to $1.67 \mathrm{fmol}$ particles. The binding capacity of DNA fragments is $3 \cdot 10^{5}$ DNA strands per particle. At 10 DNA strands per particle, the system is far from saturated. With higher concentrations of particle and DNA the reaction rate which scales $\propto[D N A][$ particle $]$ can only be expected to improve this efficiency. Therefore, the $69 \%$ efficiency is taken as the lower limit of the expected hybridization efficiency.

For a $4 \mathrm{nM}$ DNA concentration and a $16.6 \mathrm{pM}$ particle concentration an average of 170 DNA strands are bound per particle. Assuming a perfectly smooth particle, by geometrical constraint an average of $4 \%$ of the $40 \mathrm{~nm}$ DNA tethers on the particle can simultaneously interact with the substrate, which means an average of 6.8 DNA strands available for binding to the surface. This number increases to 34 DNA strands for particles functionalized with $20 \mathrm{nM}$ DNA. The number of actually formed tethers depends on the local availability of TexasRed binding antibodies on the surface, therefore also the antibody incubation conditions should be taken into account. 


\section{Number of bound particles as function of the DNA density}

The capture efficiency of particles onto the surface depends on the number of DNA targets and the number of antibody capture molecules on the substrate. The number of surface bound particles was determined for various concentrations of biomolecules. The system was prepared using the protocol described in the paper.

a

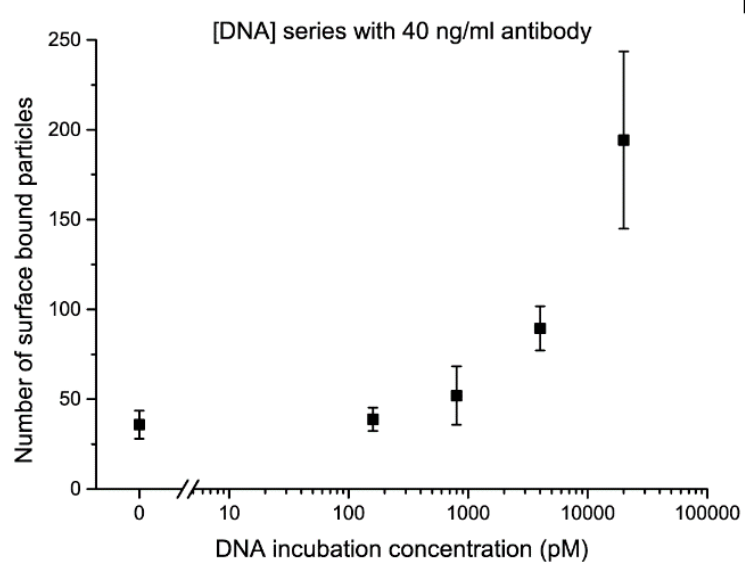

b

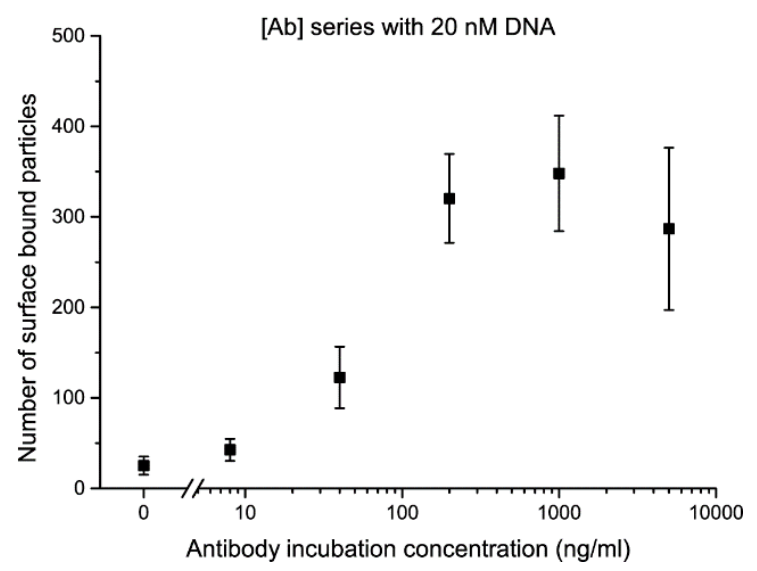

Figure S3: bound number of particles as function of the binding molecules. Number of surface bound particles as function of the a) DNA incubation concentration for a surface incubated with $40 \mathrm{ng} / \mathrm{ml}$ TexasRed antibodies and b) Antibody incubation concentration with particles functionalized with $20 \mathrm{nM}$ DNA. The number of bound particles refers to the average number of particles attached to the surface in a single field of view. The error bars indicate the standard deviation between the number of bound particles observed in each field of view $(N=5)$.

The number of found particles as function of the DNA incubation is shown for a surface incubated with $40 \mathrm{ng} / \mathrm{ml}$ antibodies is shown in figure S3a. Even in absence of DNA strands a small number of particles is observed to bind to the surface, this indicates that additional interactions lead to the binding of particles on the substrate. In presence of DNA the number of bound particles increases with the DNA concentration as the capture rate of particles onto the surface increases. The number of bound particles does not yet seem to saturate at the highest concentrations indicating that the system is still limited by the capture rate of particles onto the substrate.

In the antibodies series shown in figure S3b the number of particles increases with the number of antibodies attached to the substrate up to the saturation point at $1 \mu \mathrm{g} / \mathrm{ml}$. At this concentration either the DNA coverage on the particle becomes the limiting factor or the fluid cell is depleted of particles.

These results show a healthy response of the system to the component concentrations and illustrate that the biochemistry works as expected. 


\section{Friction experienced by particles pinned to the surface by molecular bonds}

Double molecular tethering leads to stripe-shaped motion patterns. An alternative scenario that could lead to a stripe pattern occurs when a particle is in close proximity to the surface, and is provided with a pivot line that enables a rolling motion of the particle. If the particle presents two protrusions with a short tether bound in the center, the two protrusions act as pivot points. Here we estimate if the pinning force of the tethers could lead to sufficient friction to prevent the particle from rotating the line of motion through Brownian rotation.

Pinning down of the particle can occur when the effective tether length is short, for example due to an indentation in the particle surface. The pinning of the particle by the tether would need to be strong enough to have friction forces prevent rotation of the particle with respect to the surface. The clamping force of a stretched DNA strand can be estimated using the relation ${ }^{1}$ :

$$
F(x)=\frac{k_{b} T}{P}\left(\frac{1 / 4}{\left(1-x / L_{o}\right)^{2}}-\frac{1}{4}+\frac{x}{L_{0}}\right)
$$

in which $P$ is the DNA persistence length, $T$ the temperature and $L_{0}$ the DNA contour length. For example, the energy required to stretch the $40 \mathrm{~nm}$ DNA strand $39 \mathrm{~nm}$ is given by the integral $\int_{0 \mathrm{~nm}}^{39 \mathrm{~nm}} F(x) d x$. This equals to $8 k_{b} T$ in energy, making it occur with a probability less than $10^{-3}$. In that configuration the clamping force of the DNA strand $F(39 \mathrm{~nm})=35 p N$. Surface friction forces are given by $F_{f}<\mu_{\text {friction }} \cdot F_{n}$ in which the friction coefficient is generally below unity, which limits the friction forces to an upper limit of $35 \mathrm{pN}$. The hydrodynamic drag acting on a particle rotating with $\frac{1}{2} k_{b} T$ rotational energy is $55 \mathrm{pN}$ when projected to act on the perimeter of the particle and does not rotationally confine the particle. Surface friction forces are by comparison with the drag forces also too low to confine the particle rotationally. This mechanism is therefore unlikely to contribute to the experimentally observed stripe patterns. 


\section{Verification of the implementation of the Monte Carlo simulation method}

The Monte Carlo simulation method uses the worm-like-chain method to simulate the DNA strands. The implementation of the worm-like-chain method is verified by comparing simulation results with theoretical solutions of the model.

In the worm-like-chain model there is a correlation between the orientation of the DNA strand along its controur length. This is described by the formula $\left\langle\vec{t}(s) \cdot \vec{t}\left(s^{\prime}\right)\right\rangle=e^{\left|s-s^{\prime}\right| / P}$ in which the $\vec{t}(s)$ is the orientation vector of the DNA strand at position $s$ along the contour tether and $P$ is the persistence length. A total of $5 \cdot 10^{4}$ simulations of DNA strands of $250 \mathrm{~nm}$ in length with a persistence length of $50 \mathrm{~nm}$ are made and the average correlations along the DNA strands is measured. The used segment length is $1 \mathrm{~nm}$.

The end-to-end distance $R$ of a strand of DNA depends on the contour length $L_{c}$ and the persistence length $P$ and is given by the relationship $\left\langle R^{2}\right\rangle=2 P L_{c}-2 P^{2}\left(1-e^{-L_{c} / P}\right)$. For DNA strands with a contour length ranging from 25 to $500 \mathrm{~nm}$ with a persistence length of $50 \mathrm{~nm}$ the mean squared average end-to-end distance is determined from $1 \cdot 10^{3}$ simulations.

The resulting comparison between theory and simulated results is given in figure S3. An excellent agreement is found between simulated results and theoretical expected values, confirming the proper implementation of the worm-like-chain in the Monte Carlo simulation method.

Persistence length correlation

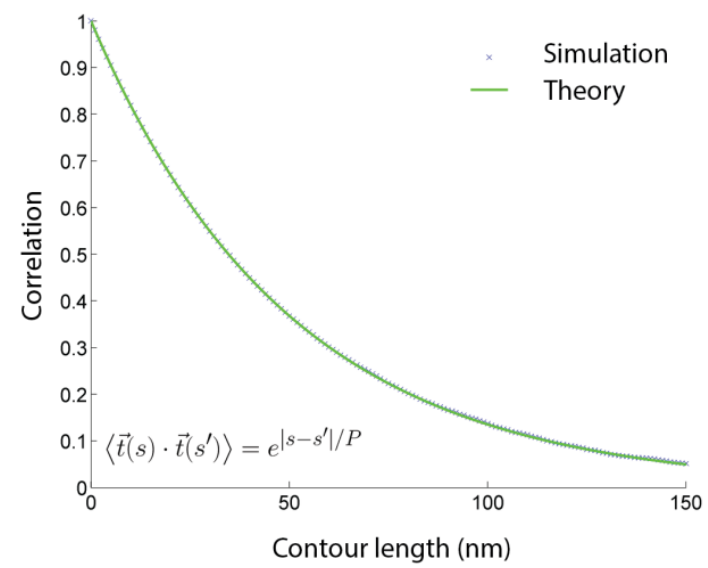

DNA end-to-end distance

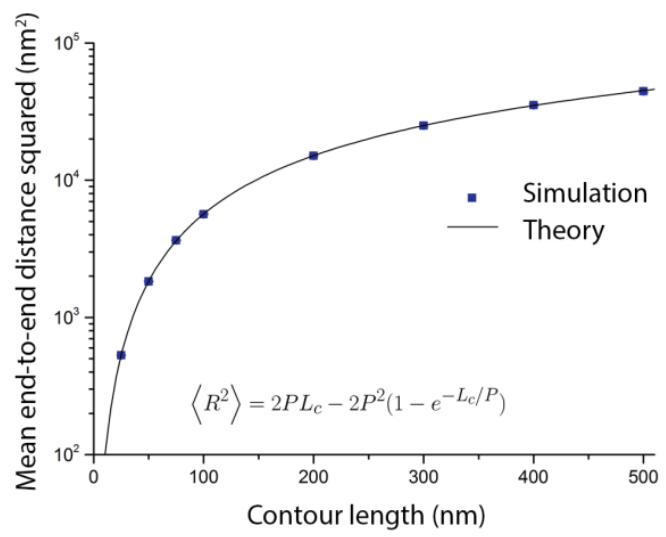

Figure S4: Comparison of simulations of and theoretical solutions to the worm-like-chain model. Left panel: simulated correlation in the orientation of the DNA strand compared to the theoretical expected correlation as a function of the distance along the DNA strand. The simulated graph is calculated from $5 \cdot 10^{4}$ simulations of DNA strands $250 \mathrm{~nm}$ in length. Right panel: simulated end-to-end distances of DNA strands compared to theoretical solutions. Every simulated data point is determined from $1 \cdot 10^{3}$ simulated DNA strands.

\section{Additional simulations of particle roughness}

A spherical protrusion on a (spherical) particle can be characterized by three parameters. In addition to how far the protrusion extends from the particle, also the diameter of the protrusion and the position of the protrusion relative to the tether attachment point are relevant. These parameters are 
denoted as $P, D$ and $\theta$ respectively. $P$ describes how far the protrusion protrudes from the particle's surface. $D$ is defined as the geometrical diameter of the particle. $\theta$, which defines the protrusion's position relative to the tether point, is chosen as the angle between the two lines connecting the particle's center with the tether attachment point and the center of the protrusion respectively. The parameters are defined in figure S4a.

Each of these parameters changes the protrusion in the system configuration and therefore leads to a different motion pattern. The particle and tether properties also influence the behavior of the system. The particle's diameter in the simulations is $1 \mu \mathrm{m}$, the tether length is $40 \mathrm{~nm}$ with a persistence length of $50 \mathrm{~nm}$, which corresponds to the experimental system. We performed simulations in which each of the three defined parameter is systematically varied while keeping the other parameters constant. The simulated motion patterns are shown in figure S4b.

a

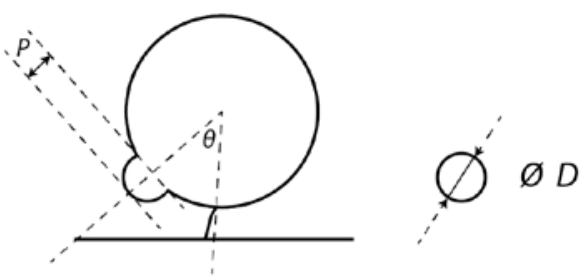

b

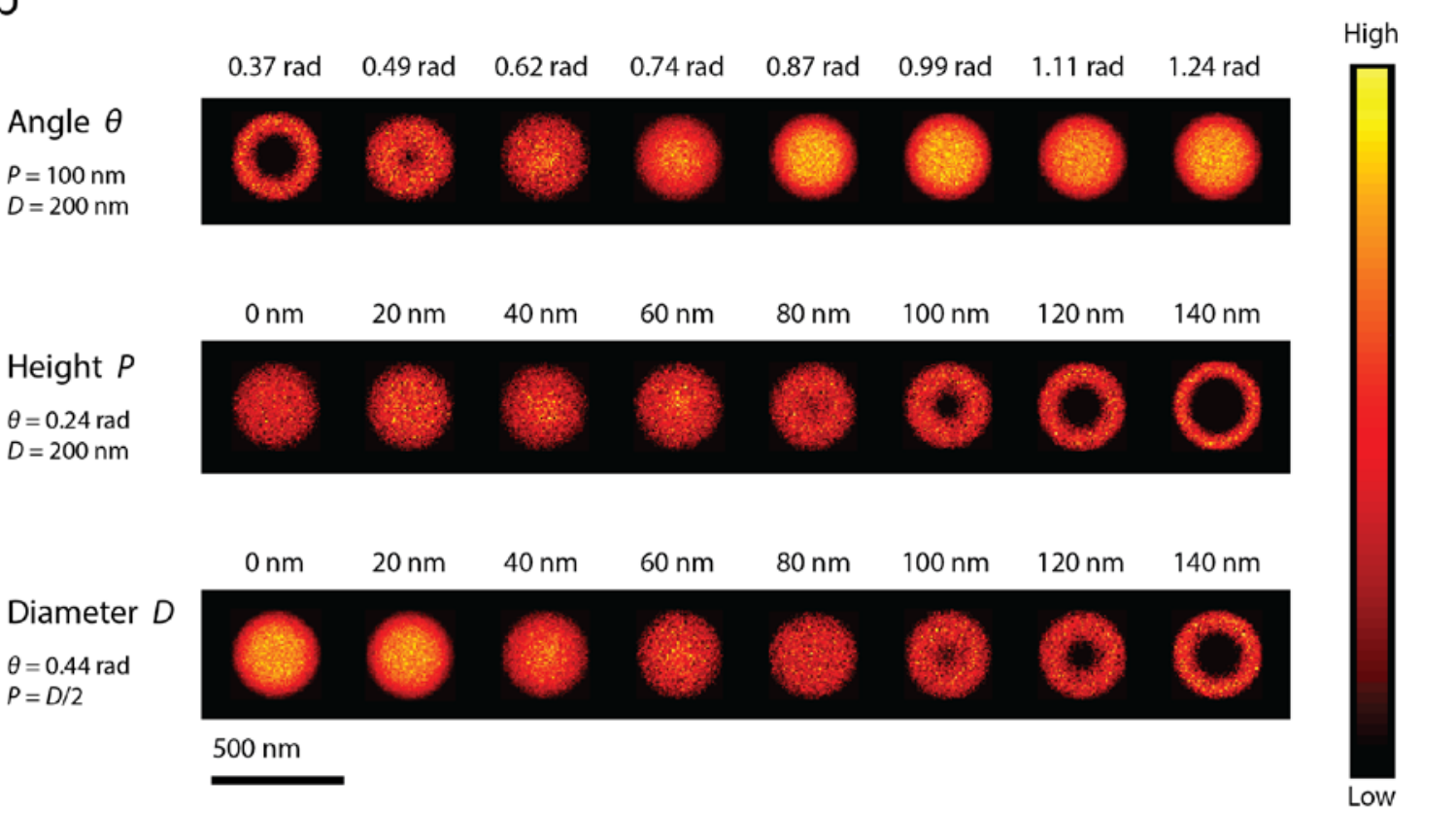

Figure S5: Simulated motion patterns for tethered particles affected by different protrusions. (a) Sketch of the system geometry and definition of the parameters $P, D$ and $\theta$. The particle has a diameter of $1 \mu \mathrm{m}$ and a $40 \mathrm{~nm}$ tether with a persistence length of $50 \mathrm{~nm}$. (b) Resulting motion patterns when each of the three parameters is systematically varied. The motion patterns are represented by a map of the probability of observing a particle at a certain location. The probability is represented by the linear color map shown on the right.

In each of the three simulation series we observe a gradual change from a disc shaped motion pattern to a ring shaped motion pattern. This seems to indicate that each of the parameters leads to a similar change in the geometry and thus similar effect on the motion pattern. It is therefore likely that the protrusion strength can be described by a single parameter. 


\section{Model describing the tethering of particles to the surface by target molecules}

Here we describe the model that estimates the number of particles bound to the substrate through a single, double or more bonds. The system contains a number of particles $N_{\text {particles }}$, the particles have a radius $D$, and they are bound to the substrate via a tether with length $l$. The fraction of the particle surface on which the tethers can interact simultaneously with the surface is $f=l / D$ for $l<D / 2$. This fraction of the particle surface is called a surface patch.

As the tether permits free rotation of the particle around the tether the surface available for binding is larger than the maximum surface available at any instant. DNA strands are dynamically bending molecules, which gives them a shorter average length, slightly mitigating this effect. When a particle is bound by two tethers, the available binding surface is again reduced as rotation and motion of the particle is confined by both tethers. Coarse grained molecular simulations can account for these effects in detail, but are computationally much more expensive. Therefore, we assume in our model that the effective available surface area for binding is a fixed fraction $f=l / D$ in which $l$ can be interpreted as the effective maximum length of a static bond.

The number of surface patches on a particle equals $n_{\text {patches }}=D / l$. The total number of patches in the system is:

$$
N_{\text {patches }}=N_{\text {particles }} \cdot n_{\text {patches }}
$$

All target molecules are distributed among these patches. The chance to select a single one of these patches is $q=1 / N_{\text {patches }}$. The probability that a patch has captured $N_{\text {captured }}$ target molecules as function of the number of target molecules $N_{\text {targets }}$ in the system is

$$
p\left(N_{\text {captured }}, N_{\text {targets }}\right)=q^{N_{\text {captured }}} \cdot(1-q)^{N_{\text {targets }}-N_{\text {captured }}} \cdot\left(\begin{array}{c}
N_{\text {targets }} \\
N_{\text {captured }}
\end{array}\right)
$$

The chance that a particle captures no target molecules is $P_{0}=p\left(0, N_{\text {targets }}\right)^{n_{\text {patches }}}$. And consequently the chance that a particle did capture one or more target molecules is $P_{1+}=1-P_{0}$.

We assume that all particles that did capture a target will bind to the surface. The number of targets bound to the binding patch determines the number of tethers formed between the particle and the surface. The assumption is made that the reactivity between a patch and the substrate does not depend on the number of targets on that patch, e.g., a patch with one target is equally reactive as a patch with ten targets.

The fraction of total particles bound to the surface with $N_{\text {tethers }}$ is then calculated with

$$
f\left(N_{\text {tethers }}>0\right)=P_{1+} \cdot \frac{p\left(N_{\text {tethers }}, N_{\text {targets }}\right)}{\sum_{N_{\text {captured }}=1}^{\infty} p\left(N_{\text {captured }}, N_{\text {targets }}\right)}
$$

The number of unbound particles is $N_{\text {unbound }}=P_{0} \cdot N_{\text {particles }}$. The number of particles bound through $N_{\text {tether }}$ target molecules is $N_{N_{\text {tethers }}}=f\left(N_{\text {tethers }}\right) \cdot N_{\text {particles }}$.

(1) Marko, J. F.; Siggia, E. D. Stretching DNA. Macromolecules 1995, 28, 8759-8770. 